\title{
Transfusão de Eritrócitos em Crianças Internadas em Unidade de Terapia Intensiva Pediátrica*
}

\author{
Red Blood Cell Transfusion in Children Admitted \\ in a Pediatric Intensive Care Unit.
}

\author{
Nelise Luciano Marvulo ${ }^{1}$, Rossano César Bonatto², Mário Ferreira Carpi², \\ Sandra Mara Queiroz Ricchetti ${ }^{3}$, Marcos Aurélio de Moraes ${ }^{3}$, José Roberto Fioretto 4 .
}

\section{RESUMO}

JUSTIFICATIVA E OBJETIVOS: As indicações de transfusão de eritrócitos não estão bem estabelecidas em crianças gravemente enfermas. O objetivo deste estudo foi descrever a prática da transfusão de eritrócitos na UTI Pediátrica do Hospital de Clínicas da Universidade Estadual Paulista (HC-UNESP).

MÉTODO: Estudo retrospectivo observacional realizado durante o ano de 2003.

RESULTADOS: Setenta e cinco pacientes receberam transfusão, havendo registro de 105 indicações. Mais da metade dos pacientes $(53,3 \%)$ tinha menos que um ano de idade. Taquipnéia (75,2\%), palidez (65,7\%) e hipotensão $(51,4 \%)$ foram os registros mais freqüentemente observados antes da transfusão. Além disso, a gasometria evidenciou acidose metabólica $(68,08 \%)$ e hipoxemia $(63,8 \%)$. Dos 93 registros de valores de hemoglobina ( $\mathrm{Hb}), 54(58,1 \%)$ estavam entre 7 e $10 \mathrm{~g} / \mathrm{dL}$ e dos 90 registros de hematócrito $(\mathrm{Ht})$ observou-se que

\footnotetext{
1. Graduanda da Faculdade de Medicina de Botucatu-UNESP

2. Professor Assistente Doutor do Departamento de PediatriaUNESP; Médico Diarista da UTI-Pediátrica da UNESP

3. Médico Diarista da UTI-Pediátrica da UNESP

4. Livre Docente do Departamento de Pediatria-UNESP, Chefe da UTI-Pediátrica.
}

*Recebido da UTI Pediátrica do Hospital de Clínicas da Universidade Estadual Paulista (HC-UNESP), Botucatu, SP.

Apresentado em 05 de setembro de 2006

Aceito para publicação em 04 de dezembro de 2006

Endereço para correspondência:

Departamento de Pediatria-UNESP

Distrito de Rubião Júnior

18618-000 Botucatu, SP

Fone: (14) 3811-6274

E-mail: jrf@fmb.unesp.br

(C)Associação de Medicina Intensiva Brasileira, 2006
$66(73,3 \%)$ apresentavam valores entre $21 \%$ e $30 \%$. As principais indicações de transfusão foram anemia em 75 crianças $(71,4 \%)$ e sangramento ativo em 26 $(24,7 \%)$. O valor médio de $\mathrm{Hb}$ antes da transfusão foi de $7,82 \pm 2,82 \mathrm{~g} / \mathrm{dL}$. Sete transfusões foram indicadas para pacientes com valores de $\mathrm{Hb}>10 \mathrm{~g} / \mathrm{dL}$, crianças estas em pós-operatório imediato de intervenção cirúrgica cardíaca e casos de choque séptico.

CONCLUSÕES: A transfusão de eritrócitos vem sendo utilizada criteriosamente, com indicações restritivas (Hb entre 7 e $10 \mathrm{~g} / \mathrm{dL}$ ). Nem sempre há anotação dos valores de $\mathrm{Hb}$ imediatamente antes da transfusão. A partir deste estudo, foi elaborado um protocolo de indicação de transfusão na unidade.

Unitermos: anemia, crianças, transfusão de eritrócitos, UTI-Pediátrica.

\section{SUMMARY}

BACKGROUND AND OBJECTIVES: Indications of red blood cell transfusion in critically ill children are not very well determined. This study aims to describe red blood cells transfusion practice at the PICU of UNESP-Botucatu Medical School.

METHODS: Retrospective observational study of all patients who received transfusion during 2003.

RESULTS: Seventy five patients received transfusion and 105 indications were recorded. $53.3 \%$ of the patients were less than one year of age. Increased respiratory rate $(75.2 \%)$, paleness $(65.7 \%)$, and hypotension (51.4\%) were the alterations more frequently recorded, before transfusion. Also, metabolic acidosis $(68.08 \%)$ e and hipoxemia $(63.8 \%)$ were very frequently observed. From 93 hemoglobin $(\mathrm{Hb})$ values recorded, 54 (58.1\%) varied from 7 to $10 \mathrm{~g} / \mathrm{dL}$ and from 90 records of hematocrit $(\mathrm{Ht}) 66(73.3 \%)$ varied from $21 \%$ to $30 \%$. The main indications of the transfusion were anemia, in 75 children (71.4\%), and active bleeding in 26 (24.7\%). 
The mean value of $\mathrm{Hb}$ before transfusion was $7.82 \pm$ $2.82 \mathrm{~g} / \mathrm{dL}$. Seven transfusions were indicated for patients with $\mathrm{Hb}$ levels higher than $10 \mathrm{~g} / \mathrm{dL}$ (postoperative heart surgery and septic patients).

CONCLUSIONS: red blood cells transfusion is carefully prescribed at the PICU by using restrictive indications ( $\mathrm{Hb}$ between 7 and $10 \mathrm{~g} / \mathrm{dL})$. Not always is possible to find out records of the $\mathrm{Hb}$ levels immediately before transfusion. Hence, a protocol to better prescribe red blood cell transfusion at the PICU was adopted.

Key Words: anemia, children PICU, red blood cell transfusion

\section{INTRODUÇÃO}

A transfusão de células vermelhas é prática essencial e freqüentemente utilizada em ambiente hospitalar ${ }^{1}$. No entanto, estudos recentes associam a transfusão de eritrócitos com maior freqüência de desenvolvimento de falência múltipla de órgãos e pior prognóstico em pacientes politraumatizados, independentemente de outros índices, inclusive da gravidade do trauma ${ }^{2,3}$. Pacientes que recebem transfusão de sangue são mais propensos a terem sua internação prolongada quando comparados com aqueles que não são submetidos a este tipo de procedimento. Além disso, a transfusão de derivados do sangue é tida como indutora de imunossupressão ${ }^{4}$. Há, também, relatos de que a exposição a leucócitos halogênicos nas transfusões pode desencadear resposta do sistema imune do receptor, com aumento do risco de infecção, recorrência precoce de malignidade e aumento da mortalidade ${ }^{5}$.

A prática da transfusão de eritrócitos em pacientes gravemente enfermos exemplifica o fato de que a causa primária de erros médicos é o resultado de modelos antiquados de tratamento em meio à complexidade da Medicina moderna. O aumento da mortalidade e lesões teciduais relacionados à anemia sustenta até hoje a regra "10/30" (taxas mínimas aceitáveis: hemoglobina de $10 \mathrm{~g} / \mathrm{dL}$ e hematócrito de $30 \%$ ) proposta por Adam e Lundy, há mais de 60 anos $^{6}$, mesmo com evidências sugerindo que a transfusão pode, na verdade, piorar a evolução dos pacientes. É diante deste dilema que se criou uma confusão na determinação de valores mínimos de hematócrito $(\mathrm{Ht})$ e hemoglobina $(\mathrm{Hb})$ que podem ser aceitáveis para os pacientes graves ${ }^{3,4}$.

Freqüentemente, as transfusões de eritrócitos são indicadas sem completo entendimento dos riscos e be- nefícios do procedimento, sendo baseadas em limites pessoais ao invés de levarem em conta indicações fisiológicas e protocolos preestabelecidos. Também, a idade e co-morbidades parecem ter pouca influência na prática transfusional, sendo a presença ou não de anemia o principal fator determinante da transfusão. Dados recentes sugerem que muitos pacientes internados em UTI toleram níveis de $\mathrm{Hb}$ abaixo de $7 \mathrm{~g} / \mathrm{dL}$ e que uma estratégia transfusional de eritrócitos liberal leva a piores resultados clínicos. No entanto, este gatilho de $7 \mathrm{~g} / \mathrm{dL}$ de $\mathrm{Hb}$ para indicação de transfusão não é utilizado na prática, sendo que a maioria dos médicos intensivistas prefere adotar o antigo gatilho de $10 \mathrm{~g} / \mathrm{dL}^{4}$.

Há poucos relatos de indicações de transfusão de derivados do sangue para crianças internadas em UTIPediátrica (UTIP) ${ }^{2}$. Estudo transversal realizado com intensivistas pediátricos demonstrou importante variação nos protocolos de atendimento e que o valor mínimo da concentração de $\mathrm{Hb}$ escolhido como gatilho para a transfusão variou de 7 a $13 \mathrm{~g} / \mathrm{dL}$. Também foi identificado que o volume de eritrócitos administrado não estava relacionado com o valor da concentração de $\mathrm{Hb}$, sugerindo que a transfusão não havia sido adequadamente indicada ${ }^{2}$. Recentemente, Hebert e col. ${ }^{4}$ e Corwin e col. ${ }^{5}$ relataram que menos de $20 \%$ das UTI apresentam protocolo institucional próprio para a prática da transfusão, reforçando a ampla variação dos critérios de indicação transfusional.

Assim, o objetivo do estudo foi avaliar a prática da transfusão de eritrócitos na UTIP do Hospital das Clínicas da Faculdade de Medicina de Botucatu-UNESP, os critérios utilizados na indicação deste procedimento e avaliar a necessidade da implantação de protocolo específico para indicação de transfusão de eritrócitos na unidade.

\section{MÉTODO}

O estudo foi aprovado pelo Comitê de Ética em Pesquisa da Faculdade de Medicina de Botucatu-UNESP, sendo respeitadas a confidencialidade dos resultados e a não identificação dos pacientes.

Foi realizado estudo retrospectivo observacional, sendo os dados obtidos a partir de prontuários de pacientes internados na UTIP e que foram submetidos à transfusão de eritrócitos no período de janeiro a dezembro de 2003. Foram incluídas crianças com idade entre 28 dias e 14 anos, casos estes selecionados com base no cruzamento de dados dos registros de saída 
de bolsas de sangue do Hemocentro do Hospital das Clínicas-UNESP e dos registros de pacientes internados na UTIP. Os pacientes transfundidos (PTs) foram aqueles que receberam pelo menos uma transfusão de eritrócitos durante a internação na UTIP e as crianças que não receberam transfusão (PNTs) foram separadas para comparação com relação a idade, sexo, diagnósticos de internação e escore Pediatric Risk of Mortality (PRISM) à admissão.

Para registro dos dados foi elaborado um protocolo de pesquisa que continha: dados de identificação; diagnóstico principal; dados clínicos registrados no dia da transfusão; dados laboratoriais no dia da transfusão; indicação da transfusão; número de transfusões e total de infusão; presença ou não de reação transfusional; tempo de internação na UTIP e evolução do paciente. Os dados foram coletados por graduanda do $4^{\circ}$ ano do Curso de Medicina. Antes da coleta, ela reviu temas de Medicina intensiva pediátrica, principalmente os artigos sobre transfusão de hemocomponentes em crianças internadas em UTIP. Além disso, elaborou o protocolo e o aplicou, inicialmente, de forma experimental em 10 casos. $O$ treinamento permitiu segurança para obtenção dos dados e, ainda assim, se existissem dúvidas elas seriam sanadas em duas reuniões semanais com o orientador do projeto.

Hipotensão, taquicardia, taquipneia, perfusão periférica lentificada e acidose metabólica foram definidas de acordo com a conferência de Consenso Internacional sobre Sepse em Pediatria publicada em 2005, a qual explicita os valores normais para estas variáveis biológicas de acordo com a faixa etária. Hipoxemia foi definida por pelo menos um dos seguintes critérios, independendo do nível de $\mathrm{FiO}_{2}$ : a) $\mathrm{SaO}_{2}<90 \%$ e b) $\mathrm{PaO}_{2}<60 \mathrm{mmHg}^{7}$, hipercapnia por $\mathrm{PaCO}_{2}>45 \mathrm{mmHg}$ e anemia por um valor de $\mathrm{Hb}<12 \mathrm{~g} / \mathrm{dL}$. Cianose foi definida como coloração azulada da pele, lábios, língua e leito ungueal.

\section{Análise Estatística}

A análise da distribuição das freqüências de sexo e diagnósticos à internação foi realizada utilizando-se o teste de Goodman para contrastes entre e dentro de populações multinomiais. A comparação dos pacientes transfundidos e não transfundidos quanto ao escore PRISM foi realizada pelo teste $t$ de Student. Dados descritivos foram apresentados em número absoluto e/ou porcentagem. Os resultados foram discutidos no nível de significância de $5 \%$.

\section{RESULTADOS}

No período eleito para o estudo, 250 crianças foram admitidas na UTIP. Destas, 75 (30\%) receberam transfusão de células vermelhas durante a internação. Não foi possível analisar os registros de 10 pacientes, alguns por não localização do prontuário e outros por ausência de anotações sobre a transfusão. Como os pacientes poderiam receber mais de uma transfusão, foram registradas 105 (42\%) indicações de transfusão sanguínea, com média de 1,6 indicações por paciente. Quarenta e dois pacientes receberam apenas uma transfusão e um paciente foi submetido a seis transfusões, como ilustrado na figura 1.

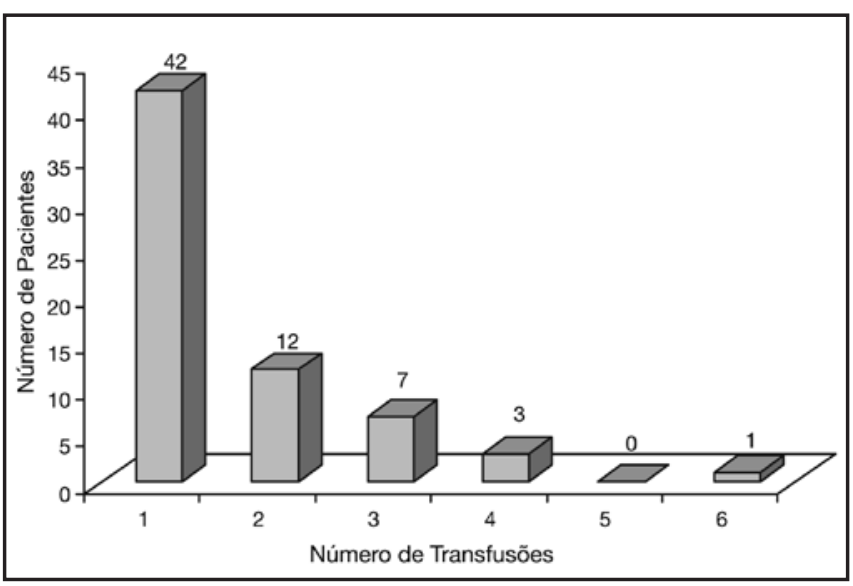

Figura 1 - Número de Transfusões Administradas para cada Paciente

A tabela 1 ilustra a caracterização dos casos quanto ao sexo, distribuição etária e escore PRISM.

Tabela 1 - Caracterização da População quanto ao Sexo, Idade e Escore PRISM.

\begin{tabular}{lccc}
\hline & $\begin{array}{c}\text { Todos os Casos } \\
(\mathrm{n}=250)\end{array}$ & $\begin{array}{c}\text { PTs } \\
(\mathrm{n}=75)\end{array}$ & $\begin{array}{c}\text { PNTs } \\
(\mathrm{n}=175)\end{array}$ \\
\hline $\begin{array}{l}\text { Sexo (feminino) } \mathrm{n}(\%) \\
\text { ldade, } \mathrm{n}(\%)\end{array}$ & $128(51,2)$ & $39(52)$ & $89(50,8)$ \\
$\quad$ < 1 ano & $138(55,2)$ & $40(53,3)$ & $95(54,2)^{*}$ \\
1 a 3 anos & $63(25,2)$ & $17(22)$ & $40(23)$ \\
3 a 5 anos & $21(8,4)$ & $06(8,57)$ & $14(8)$ \\
> 5 anos & $28(11,2)$ & $12(16,2)$ & $26(14,8)$ \\
PRISM (x \pm dp) & $9,5 \pm 6,5$ & $13,3 \pm 4,8$ & $6,5 \pm 6^{\#}$ \\
\hline
\end{tabular}

$\mathrm{n}=$ número de pacientes; PTs = pacientes transfundidos; PNTs = pacientes não transfundidos:

PRISM = Pediatric Risk of Mortality.

* $p<0,05$ na comparação entre as freqüências de pacientes com menos de um ano de idade e as demais para PTs e PNTs.

$\# p<0,05$ na comparação entre PTs e PNTs. 
Não houve diferença estatística significativa na distribuição dos pacientes quanto ao sexo quando se comparou os PTs e os PNTs. Quanto a idade, observouse que mais da metade das crianças transfundidas (53,3\%) tinham menos que um ano, sendo esta faixa etária a mais prevalente na população geral da UTIP e também nos PNTs. Levando-se em conta os 75 casos de PTs, a insuficiência respiratória aguda (IRA) foi o principal diagnóstico, presente em 29 (38\%) pacientes. Os demais diagnósticos foram, principalmente, sepse/choque séptico (14,3\%), pós-operatório imediato $(13,3 \%)$, insuficiência cardíaca congestiva $(5,7 \%)$, choque hipovolêmico $(4,76 \%)$ e outros $(23,9 \%)$. A freqüência de IRA foi significativamente maior quando comparada aos demais diagnósticos dentro do grupo de PTs, na população geral e nos PNTs $(p<0,05$ - teste de Goodman).

Registro de palidez no dia da transfusão foi observado em $65,7 \%$ dos pacientes. A pressão arterial de $15,2 \%$ dos pacientes estava dentro da normalidade, 51,4\% apresentavam hipotensão e esta variável não foi registrada em 33,3\% dos pacientes. Taquicardia foi observada em $19 \%$ dos exames pré-transfusionais. Havia taquipnéia em $75,2 \%$ dos pacientes, $40 \%$ apresentavam-se febris e $4,7 \%$ estavam hipotérmicos. Cianose foi referida em $3,8 \%$ dos casos. A perfusão periférica estava lentificada em 23 pacientes $(21,9 \%)$ e não foi encontrado registro desta variável em $31,4 \%$ dos casos.

Dos 75 pacientes, foi obtido registro de gasometria arterial imediatamente antes da transfusão em 47 crianças, havendo acidose metabólica em 32 casos $(68,08 \%)$, hipoxemia em $30(63,8 \%)$ e hipercapnia em $23(48,9 \%)$.

Os valores pré-transfusionais de $\mathrm{Ht}$ e $\mathrm{Hb}$ estão apresentados na tabela 2. Dos 93 registros de valores de
$\mathrm{Hb}, 54(58,1 \%)$ estavam entre 7 e $10 \mathrm{~g} / \mathrm{dL}$ e dos 90 registros de $\mathrm{Ht}$ observou-se que 66 (73,3\%) apresentavam valor entre $21 \%$ e $30 \%$. Não foi encontrado registro dos valores de $\mathrm{Hb}$ em 12 pacientes e de $\mathrm{Ht}$ em 15. As principais indicações de transfusão de concentrado de hemácias foram anemia em 75 crianças $(71,4 \%)$, sangramento ativo em 27 (25,7\%), hipoxemia refratária à administração de oxigênio em dois (1,9\%) e em um paciente não foi obtido registro da indicação.

$\mathrm{O}$ valor médio de $\mathrm{Hb}$ nos pacientes submetidos a transfusões de concentrados de hemácia foi de 7,82 $\pm 2,82$ $\mathrm{g} / \mathrm{dL}$. Sete transfusões foram indicadas para pacientes com valores de $\mathrm{Hb}$ superiores a $10 \mathrm{~g} / \mathrm{dL}$, sendo os diagnósticos destas crianças pós-operatório imediato de ventriculoseptoplastia e choque séptico. Não foram registradas reações transfusionais. A tabela 3 ilustra os valores médios de $\mathrm{Hb}$ dos pacientes segundo os principais diagnósticos.

A média de internação dos PTs foi de 8,7 dias, sendo que $21,9 \%$ permaneceram internados por mais do que 2 semanas. Receberam alta 73,3\%,1,9\% foram transferidos para outros serviços e $20,9 \%$ foram a óbito. A mortalidade e o tempo médio de internação da população geral da UTIP foram, respectivamente, $11,2 \%$ e 3,2 dias e para os PNTs foram de $10,2 \%$ e $4,3 \%$, respectivamente.

\section{DISCUSSÃO}

Existem poucos estudos sobre indicações de transfusão de eritrócitos em crianças gravemente enfermas, fazendo com que haja grande variação de indicações de transfusão de células vermelhas em crianças internadas em UTIP ${ }^{4,9}$.

Observou-se que a maioria destes pacientes tinha menos de um ano de idade e a sepse foi a segunda causa

Tabela 2 - Distribuição dos Pacientes segundo os Valores de Hematócrito e Hemoglobina Imediatamente antes da Transfusão.

\begin{tabular}{|c|c|c|c|c|c|c|c|c|}
\hline & \multicolumn{3}{|c|}{ Hemoglobina (g/dL) } & \multirow[t]{2}{*}{ Total } & \multicolumn{3}{|c|}{ Hematócrito (\%) } & \multirow[t]{2}{*}{ Total } \\
\hline & $<7$ & $7-10$ & $>10$ & & $<21$ & $21-30$ & $>30$ & \\
\hline Pacientes (n - \%) & $32(34,4 \%)$ & $54(58,1 \%)$ & $7(7,5 \%)$ & $93(100 \%)$ & $17(18,9 \%)$ & $66(73,3 \%)$ & $7(7,8 \%)$ & $90(100 \%)$ \\
\hline
\end{tabular}

Tabela 3 - Valores Médios de Hemoglobina, antes da Transfusão, segundo os Diagnósticos.

\begin{tabular}{lc}
\hline Diagnósticos & Valores de Hemoglobina (g/dL) \\
\hline Sangramento ativo, $\mathrm{n}=6$ & 5,3 \\
Insuficiência respiratória aguda, $\mathrm{n}=41$ & 7,2 \\
Sepse/choque séptico, $\mathrm{n}=38$ & 7,9 \\
Insuficiência cardíaca congestiva, $\mathrm{n}=6$ & 8,1 \\
Pós-operatório imediato, $\mathrm{n}=14$ & 9,1 \\
\hline
\end{tabular}


mais frequente de internação. Além disso, sangramento ativo foi uma das principais indicações de transfusão. Embora as perdas primárias de sangue por cirurgia, trauma ou sangramento gastrintestinal representem as causas mais freqüentes de anemia em pacientes internados em UTIP, as concentrações de $\mathrm{Hb}$ podem diminuir mesmo na ausência de sangramento. Resposta anormal da eritropoetina à perda de sangue determinada por mediadores inflamatórios e vasodilatação secundária à resposta inflamatória levam a aumento do volume sanguíneo, contribuindo para diminuir o Ht em quadros sépticos ${ }^{10}$. Além disso, em Pediatria, é particularmente importante e negligenciada a perda sanguínea induzida por coleta de amostras de sangue para exames laboratoriais ${ }^{5}$. No estudo Anemia and Blood Transfusion in Critical Care o volume médio diário de sangue retirado de flebotomia foi de $41 \mathrm{~mL}$, sendo significativamente maior nos pacientes sépticos ${ }^{11}$. Apesar do volume de sangue retirado de cada paciente para exames laboratoriais não ter sido avaliado, é possível que a baixa idade destes pacientes tenha contribuído para a alta freqüência de anemia na população, tanto pela retirada de amostras de sangue como pela carência de ferro. A primeira tem maior impacto nos pacientes de mais baixa idade, da mesma forma que a carência de ferro é mais frequente nos lactentes.

No presente estudo, a transfusão de eritrócitos foi realizada em $30 \%$ de todos os pacientes admitidos na unidade. Esta porcentagem está em acordo com vários estudos que mostraram que a porcentagem de pacientes gravemente enfermos que receberam transfusão de sangue durante a internação variou de $20 \%$ a $53 \%{ }^{10}$. Estudo Canadense, envolvendo 5.298 pacientes internados em UTI, relatou que $25 \%$ dos pacientes receberam transfusão de hemácias ${ }^{12}$ e no Reino Unido a porcentagem relatada foi $53 \%$, em 1.247 pacientes avaliados ${ }^{13}$.

Na literatura há intenso debate sobre qual o "valor crítico" de Hb abaixo do qual a liberação de oxigênio para os tecidos é prejudicada e não se sabe qual nível de $\mathrm{Hb}$ deve ser utilizado para indicar a transfusão ${ }^{10}$.

Historicamente, acreditava-se que os níveis de $\mathrm{Hb}$ deveriam ser mantidos em valores acima de $10 \mathrm{~g} / \mathrm{dL}$ para que fosse garantida boa oferta de oxigênio aos tecidos. Atualmente, vários autores recomendam que as transfusões sejam realizadas diante de valores de $\mathrm{Hb}$ abaixo de $7 \mathrm{~g} / \mathrm{dL}$ e que se evite transfusões com valores acima de $10 \mathrm{~g} / \mathrm{dL}^{5,14-17}$. Estudos experimentais e clínicos relataram que o risco de morte se eleva significativamente em pacientes com valores de $\mathrm{Hb}$ entre
3,5 e $4 \mathrm{~g} / \mathrm{dL}$, taxa na qual a anemia aumenta a produção de lactato e a extração de oxigênio eleva-se a mais de $50 \%$. No entanto, o limite inferior de tolerância ainda não é conhecido. Para valores entre 6 e $10 \mathrm{~g} / \mathrm{dL}$, a indicação de transfusão de eritrócitos depende da extensão da perda sanguínea, doenças cardíacas subjacentes e estado clínico geral ${ }^{10}$.

Apesar destes dados, não é de uso corrente a utilização de protocolos para normatizar a transfusão de eritrócitos em crianças gravemente enfermas ${ }^{2,4,5}$. Na UTIP, tem-se procurado indicar transfusão de eritrócitos adotando critério mais restritivo, sem, no entanto, utilizar protocolo específico. Apesar disso, foram observados que o valor médio de $\mathrm{Hb}$ dos pacientes antes da transfusão foi de $7,82 \pm 2,82 \mathrm{~g} / \mathrm{dL}$. Estudos semelhantes relataram valores de $\mathrm{Hb}$ de $8,6 \pm 1,7 \mathrm{~g} / \mathrm{dL}^{1,5}$. Acrescenta-se que sete transfusões foram indicadas para pacientes com valores de $\mathrm{Hb}$ superiores a $10 \mathrm{~g} /$ dL. No entanto, a análise dos prontuários evidenciou que eram pacientes em pós-operatório imediato de cirurgia cardíaca e distúrbio de coagulação e crianças em choque séptico, com distúrbio hemodinâmico e em uso de fármacos vasoativos. Estas condições clínicas podem justificar a indicação mais liberal nestes casos. Igualmente, indicações mais liberais em pacientes com diagnósticos semelhantes foram relatadas por Laverdière e col. ${ }^{2}$ e por Armano e col. ${ }^{7}$. Armano e col. ${ }^{7}$ caracterizaram como determinantes da transfusão de células vermelhas em crianças internadas em UTIP a presença de anemia, doença cardíaca, sepse e disfunção de múltiplos órgãos. Para os pacientes com sepse, no entanto, a oferta de oxigênio está aquém da demanda tecidual e a transfusão de concentrado de hemácias só é eficaz para aumentar a oferta de oxigênio quando o consumo não estiver limitado pelo conteúdo sanguíneo de oxigênio ou sua capacidade de transporte ${ }^{18}$. Existem evidências de que disfunções sistêmicas na microcirculação ${ }^{19}$ associadas à menor capacidade das hemácias transfundidas sofrerem alterações em seu formato podem trazer prejuízos consideráveis aos pacientes com sepse ${ }^{20,21}$.

Interessante foi a observação de que os valores do escore PRISM foram significantemente mais elevados nos PTs, em acordo com Armano e col. ${ }^{7}$ que demonstraram que o escore PRISM é determinante da indicação de tranfusão, além dos já descritos.

O tempo de internação dos PTs foi maior que o descrito por outros estudos onde a permanência em UTI foi mais curta (média de 7,4 dias de internação) ${ }^{5}$. Sabe-se que o número de pacientes que recebem transfusão 
aumenta de acordo com o período de internação ${ }^{5}$, podendo este fator explicar a freqüência de transfusão de eritrócitos de $30 \%$ observada neste estudo. Acrescenta-se que esta freqüência está em acordo com outros relatos da literatura, como já referido.

A literatura mostra que em $4 \%$ das transfusões pode haver reação transfusional ${ }^{5}$. Não se observou nenhum caso de reação transfusional, sendo que tal fato pode ser explicado pelos cuidados na preparação e na administração dos produtos do sangue adotados na instituição.

Limitações do Estudo

O estudo tem como principal limitação seu desenho retrospectivo observacional, o qual faz com que possa ter ocorrido perda de informações e/ou que algumas delas não sejam absolutamente corretas. Além disso, todos os protocolos foram preenchidos por uma graduanda do Curso de Medicina, a qual, apesar de treinamento prévio, pode ter perdido algum dado importante. Por outro lado, há um ponto favorável. O descompromisso da aluna com um ou outro resultado permitiu que os dados fossem anotados como aparecem nos prontuários.

\section{CONCLUSÕES}

1. Apesar de não haver protocolo específico, o serviço tem indicado transfusão de eritrócitos utilizando critérios mais restritivos, ou seja $\mathrm{Hb}$ entre 7 e $10 \mathrm{~g} / \mathrm{dL}$; 2. Nem sempre há anotação dos valores de $\mathrm{Hb}$ e Ht imediatamente antes da transfusão.

A partir deste estudo, a equipe elaborou e instituiu protocolo de indicação de hemoderivados na unidade para permitir registro de todos os dados e acompanhamento mais adequado dos casos, limitando e adequando cada vez mais a transfusão de sangue na UTI-Pediátrica.

\section{REFERÊNCIAS}

01. Hebert PC, Fergusson DA - Red blood cell transfusions in critically ill patients. JAMA, 2002;288:1525-1526.

02. Laverdiere C, Gauvin F, Hebert PC et al - Survey on transfusion practices of pediatric intensivists. Pediatric Crit Care Med, 2002;3:335-340.

03. Shah JS, Hickey R - Anemia and blood transfusion in the critically ill: a decade without change. Crit Care Med, 2004;32:290-291.

04. Hebert PC, Yetisir E, Martin C et al - Is a low transfusion threshold safe in critically ill patients with cardiovascular diseases? Crit Care Med, 2001;29:227-234

05. Corwin HL, Gettinger A, Pearl RG et al - The CRIT Study: Anemia and blood transfusion in the critically ill - current clinical practice in the United States. Crit Care Med, 2004;32:39-52.

06. Adam RC, Lundy JS - Anesthesia in cases of poor risk: some suggestions for decreasing the risk. Surg Gynecol Obstet, 1942;74:1011-1101.

07. Armano r, Gauvin F, Ducruet T, et al - Determinants of red blood cell transfusion in a pediatric critical care unit: A prospective, descriptive epidemiological study. Crit Care Med, 2005;33:2637-2644.

08. Goldstein B, Giroir B, Randolph A et al - International pediatric sepsis consensus conference: definitions for sepsis and organ dysfunction in pediatrics. Pediatr Crit Care Med, 2005;6:2-8.

09. Corwin HL, Surgenor SD, Gettinger A - Transfusion practice in the critically ill. Crit Care Med, 2003;31:(Suppl):S668-S671.

10. Vincent JL, Piagnerelli M - Transfusion in the intensive care unit. Crit Care Med, 2006;34:(Suppl5):S96-S101.

11. Vincent JL, Baron JF, Reinhart $\mathrm{K}$ et al - Anemia and blood transfusion in critically ill patients. JAMA, 2002;288:1499-1507.

12. Hebert PC, Wells G, Martin C et al - Variation in red cell transfusion practice in the intensive care unit: a multicentre cohort study. Crit Care, 1999;3:57-63.

13. Rao MP, Boralessa $\mathrm{H}$, Morgan $\mathrm{C}$ et al - Blood component use in critically ill patients. Anaesthesia, 2002;57:530-534

14. Ferreira JS, Ferreira VLPC, Pelandre GL - Red blood cell transfusion in the Intensive Care Unit. Rev Bras Hematol Hemoter, 2005;27:179-182.

15. Walsh TS, Garrioch M, Maciver $\mathrm{C}$ et al - Red cell requirements for intensive care units adhering to evidence-based transfusion guidelines. Transfusion, 2004;44:1405-1411.

16. Hebert PC, Wells G, Blajchman MA et al - A multicenter, randomized, controlled clinical trial of transfusion requirements in critical care. Transfusion Requirements in Critical Care Investigators, Canadian Critical Care Trials Group. N Engl J Med, 1999;340:409-417.

17. Murphy MF, Wallington TB, Kelsey $P$ et al - Guidelines for the clinical use of red cell transfusion. Br J Haematol. 2001;113:24-31.

18. Mink RB, Pollack MM - Effect of blood transfusion on oxygen consumption in pediatric septic shock. Crit Care Med, 1990;18:1087-1091.

19. Hersch M, Gnidec AA, Bersten $A D$ et al: - Histologic and ultrastructural changes in nonpulmonary organs during early hyperdynamic sepsis. Surgery, 1990;107:397-410.

20. Langenfeld JE, Livingston DH, Machiedo GW - Red cell deformability is an early indicator of infection. Surgery 1991:110:398-404.

21. Mollitt DL, Poulos ND - The role of pentoxifylline in endotoxin-induced alterations of red cell deformability and whole blood viscosity in the neonate. J Pediatr Surg, 1991;26:572-574. 